\title{
Human papillomavirus infection increases the risk of breast carcinoma: a large-scale systemic review and meta-analysis of case-control studies
}

\author{
Chutong Ren, Kai Zeng, Chujun Wu, Lan Mu, Jiangsheng Huang, Mingming Wang \\ Department of Minimally Invasive Surgery, the Second Xiangya Hospital, Central South University, Changsha 410000, China \\ Contributions: (I) Conception and design: C Ren; (II) Administrative support: J Huang; (III) Provision of study materials or patients: C Ren, K Zeng; \\ (IV) Collection and assembly of data: C Ren, K Zeng, C Wu, L Mu; (V) Data analysis and interpretation: C Ren, C Wu; (VI) Manuscript writing: All \\ authors; (VII) Final approval of manuscript: All authors. \\ Correspondence to: Mingming Wang, MD. Department of Minimally Invasive Surgery, The Second Xiangya Hospital, Central South University, No. \\ 139 Renmin Middle Road, Changsha 410000, China. Email: mingmingw2008@csu.edu.cn.
}

\begin{abstract}
Background: Breast carcinoma (BC) is a cancer with a high morbidity rate, but the mechanisms by which it develops are never clear. There has been speculation regarding the potential relationships between breast cancer and local HPV infections for some time, and although much clinical research supports this hypothesis, some research results disprove the association. Therefore, the association is still inconclusive.

Methods: We performed the data collection by searching the database PubMed, Embase, Cochrane Library and Web of science. In addition, 22 sites were added manually. After carefully selection, the pooled odds rate of 37 included case control studies was calculated. Subgroup analysis, publication bias and trim \& fill analysis were conducted to make the result more reliable.

Results: The analysis of 37 case control studies containing 3,607 BC cases and 1,728 controls showed obviously increase of BC risk with human papillomavirus (HPV) positive [summary odds ratio (SOR) $=6.22$, $95 \%$ confidence interval 4.25 to $9.12 ; \mathrm{P}=0.0002$ ]. Subgroup analysis proved three high risk HPV types (HPV16, 18 and 33) were positively correlated to BC.
\end{abstract}

Conclusions: This systemic review and meta-analysis provide the evidence for HPV infection as a potential risk factor in $\mathrm{BC}$, while the mechanism of this hypothesis still needs further evaluation.

Keywords: Human papillomavirus (HPV); breast carcinoma (BC); case-control studies

Submitted May 21, 2019. Accepted for publication Aug 28, 2019.

doi: 10.21037 /gs.2019.09.04

View this article at: http://dx.doi.org/10.21037/gs.2019.09.04

\section{Introduction}

Breast carcinoma (BC) is not only the most common invasive malignant neoplasm in women but also the second main cause of cancer death in women (1). According to the statistics of Global Cancer Statistics 2018, it is estimated that about 2,088,849 patients were newly diagnosed with breast cancer and 626,679 patients died from breast cancer in 2018. Among neoplastic diseases, the mortality rate of BC ranked 2nd after the lung cancer worldwide (2). Since cancer cells can develop in the ducts, lobules or tissues in between, it has multiple histological types. In the last few decades, due to the raising public awareness of the disease and the wide application of screening mammogram, more patients are diagnosed in relatively early stage than before. Also, new treatment approaches such as targeted therapy and endocrine therapy have greatly increased the overall survival rate associated with the disease and the quality of patients' life. It is assumed that one in eight women in the world will develop cancer of the mammary glands (3), in which only $5-10 \%$ of all these cases are caused by genetic disorders. The remaining $90-95 \%$ of cases are connected to environmental factors and lifestyle choices (4). Therefore, 
more investigations were carried out in order to figure out different risk factors associated with BC.

The human papillomavirus (HPV) is a non-enveloped DNA virus which belongs to the papillomaviridae family with over 150 types. It is the most common sexually transmitted infection agent in the United States. Most women will get at least one type of HPV at some point in life, since the incidence percentage is as high as $80 \%(5)$. Boshart et al. was the first to theorize that HPV was one of the potential causes of cervical cancer (6). Following further investigation of HPV, scientists discovered that HPV infections cause a significant proportion of cancers worldwide, with high-risk HPV types such as HPV16 and HPV18, being associated with squamous cell carcinomas of the anogenital and oropharyngeal tract. Furthermore, some low-risk HPV types have been observed to cause genital warts and recurrent respiratory papilloma.

The association of HPV infection and BC was put forward by Band et al. in 1990. Band et al. reported that HPV could immortalize normal human mammary epithelial cells, and reduce their requirement on growth factors (7). Since 1992, an increasing number of researches has been carried out trying to investigate the relationship between HPV infection and BC. However, the conclusions of these studies were controversial. Some scientists could not find HPV DNA in breast cancer tissues as is reported by Doosti et al. (8), Bakhtiyrizadeh et al. (9) and Gannon et al. (10), while some were able to find a high prevalence rate of HPV in breast cancer tissues such as Cavalcante et al. (11). Although almost 30 years has passed since the proposition of this theory, the definite conclusion remains unknown.

The main purpose of this study is to collect the information of original studies worldwide concerning the relationship of $\mathrm{HPV}$ infections to $\mathrm{BC}$. In order to draw a scientific conclusion, we limited the study type as casecontrol studies which are considered to be more convictive than cross-sectional study. Through the assistance of a systematic literature search method, we carefully analyzed 37 related case-control studies.

\section{Methods}

\section{Register}

We successfully registered a systematic review entitled

"Human papillomavirus infection in breast cancer patients: a meta-analysis of case-control studies" on PROSPERO. The registration number is: CRD42019121723. We have been updating our information during the systematic review writing procedure.

\section{Search strategy and study selection}

We followed the Meta-analysis of Observational Studies in Epidemiology (MOOSE) (12) for performing and reporting the present meta-analysis. The MOOSE checklist is exhibited in Table S1. Two authors (C Ren and K Zeng) independently performed the literature search procedure and study selection. Two authors reached consensus on all items. Relevant articles on the association between BC and HPV infection were identified through an extensive search of the Cochrane Library, Embase, PubMed, Web of Science and clinicaltrials.gov. In PubMed, the search was performed by using the keywords: "Breast cancer" and "Papillomavirus". We defined the following medical subject headings (MeSH) terms: "papillomaviridae" and "breast neoplasms". We combined the Mesh terms and entry words when completing the searching process. The literature search contains all literature until March 2019, with no publication starting-date limitation. These search queries yielded 12 citations in The Cochrane Library, 955 citations in Embase, 308 citations in PubMed and 1149 citations in Web of science.

Studies about the relationship between BC and HPV infection were reviewed and evaluated critically for predefined eligibility criteria. Figure 1 summarized the flow of information retrieval process and the inclusion and exclusion criteria. The searching strategy in PubMed is exhibited in the supplementary I.

\section{Data extraction}

Data extraction was performed by two authors (C Ren and $\mathrm{K}$ Zeng) independently. We collected the information of authors, year of publication, area, histologic type of control, tissue type and HPV infection related data. For those articles without a description of the entire clinical data, we made additional efforts to obtain the original data by contacting the authors.

\section{Statistical analyses}

Revman 5.3 and Stata 14.0 were utilized to analysis the retrieved data. OR and $95 \%$ CI were calculated for each article. Five subgroup analysis-the histologic type of control group, tissue type and three subtypes of HPV were 
2,424 of records identified through database searching Cochrane 12, Embase 955, PubMed 308, Web of Science 1,149, ClinicalTrials.gov 0
22 of records identified through published meta-analysis

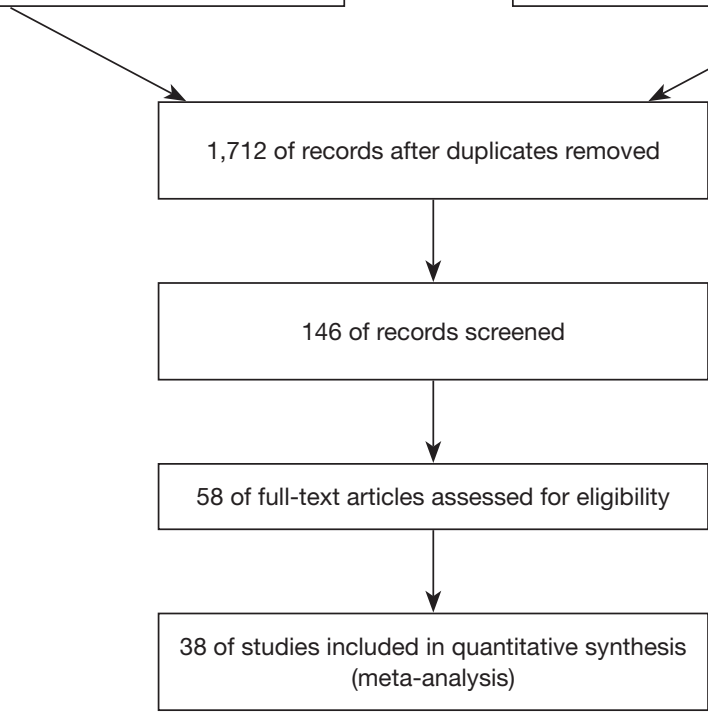

1,566 of records were excluded because of unrelated hypotheses

88 of records were excluded because of non human studies and mechanism experiment

20 of full-text articles excluded:

15 of them were excluded for case only study;

5 of them were excluded because of null results in both groups;

1 record was removed for the same specimen resource as another.

Figure 1 Flow diagram of systemic review procedure.

performed. The presence of heterogeneity in meta-analysis was evaluated using the I-squared value (\%). A P value of less than $5 \%$ was considered statistically significant. In order to estimate publication bias, Egger's linear regression and Egger's linear regression were applied. In addition, trim and fill method was applied to test if the pooled results were affected by publication bias.

\section{Results}

\section{Literature search}

Figure 1 elucidates the process of the literature search. A total of 2,424 records were identified through database searching and 22 records were manually added by going through the reference list of published meta-analysis $(13,14)$. We removed duplicates and 1,566 records by browsing titles and abstracts. Fifty-eight full-text articles were obtained for eligibility. Sixteen records were removed because of caseonly study design, and 5 were excluded because of null results in both groups, while 1 record was removed for the same specimen resource as another.

A total of 37 original studies containing 5,335 detected specimens were finally included to carry out the analysis. All the included articles were carefully assessed through reading full text. The included original studies are listed in the Table 1.

\section{Characteristics of eligible studies}

The 37 included studies range from 1999-2019 covering 17 countries worldwide. Over $50 \%$ of the research were carried out in Asian countries. Among them, 11 articles were published before 2010, 15 were published between 2010 and 2015, and 11 were published after 2015. All of the included research are case-control studies. Most studies used nonmalignant breast lesions as a control, while 9 studies used normal breast tissue as controls which are showed in Table 1 . Furthermore, we collected the statistics of HPV subtypes detection (high risk HPV subtypes HPV16, HPV18 and HPV33) in order to carry out subgroup analysis figuring out carcinogenic subtype. The specimen types used for HPV detection are as follows: 11 studies used fresh frozen tissue, 2 studies used fresh tissue, 1 study used liquid cytology specimens, and the rest used paraffin-embedded tissue.

\section{Methodological quality of included studies}

All of the 37 studies have undergone methodological quality assessment according to Newcastle-Ottawa Quality Assessment Scale designed for case control studies. The results of quality assessment are listed in Table 2. The assessment results showed that 16 original studies got 6 stars which were considered to be relatively high quality and the other 21 got 5 stars which indicated some risk of bias. 


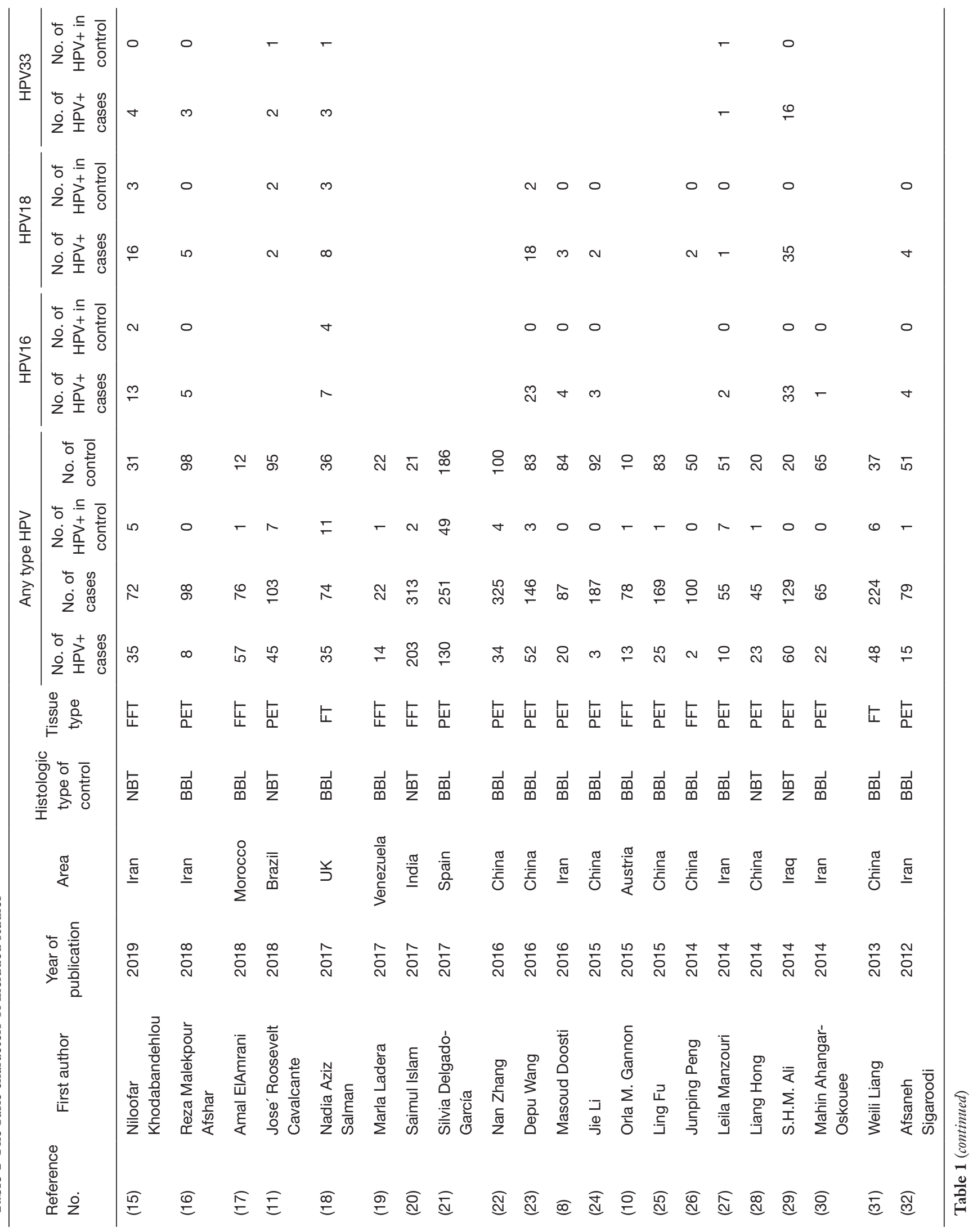




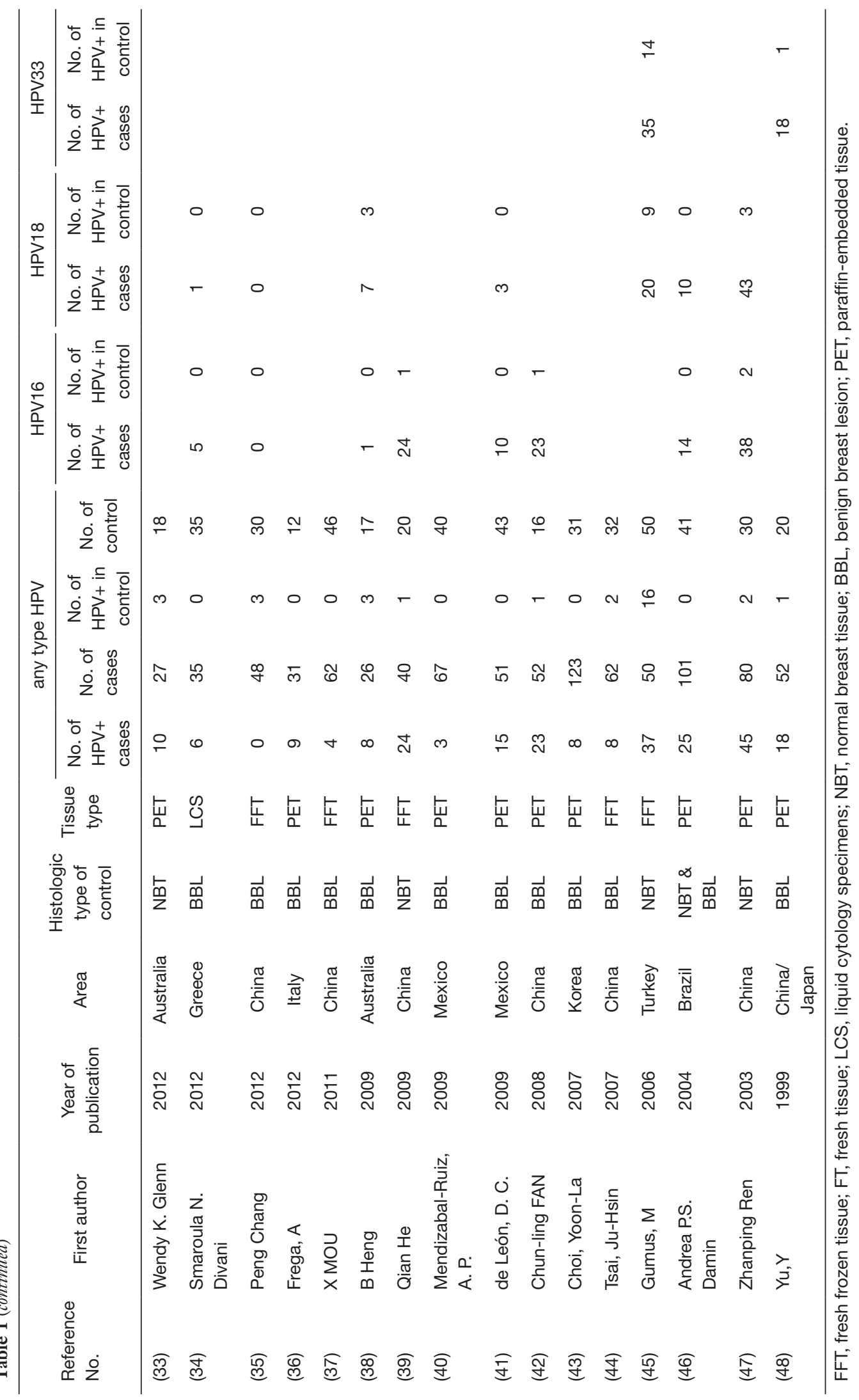




\section{$H P V$ infection and the risk of $B C$}

The results of the pooled analysis for included studies are shown in the forest plot (Figure 2). In all studies, 1,097 tissues in BC group were found HPV positive, while 132 tissues in control group were found HPV positive. Since the I-square is $52 \%$, we applied the random effect model. The summary odds ratio (SOR) was 6.22 (95\% confidence interval 4.25 to $9.12 ; \mathrm{P}=0.0002$, Figure 2) which provided evidence for the theory that HPV infection increased the risk of $\mathrm{BC}$.

\section{Subgroup analysis}

A subgroup analysis was carried to find out the cause of heterogeneity. For histologic type of control, 37 studies were divided into normal breast tissue subgroup and benign breast lesion subgroup in terms of type of control group. The SOR was 8.78 (95\% confidence interval 5.54 to 13.92 ; $\mathrm{P}<0.00001 ; \mathrm{I}^{2}=10 \%$, Figure 3) in normal breast tissue subgroup and the SOR was 4.91 (95\% confidence interval 3.08 to $7.82 ; \mathrm{P}<0.00001 ; \mathrm{I}^{2}=50 \%$, Figure 3 ) in the benign breast lesion subgroup. This subgroup analysis evidently declined the heterogeneity.

For tissue type, the original studies were divided into paraffin-embedded tissue subgroup and fresh frozen tissue subgroup. In this way, SOR was 7.43 (95\% confidence interval 4.56 to $12.09 ; \mathrm{P}<0.00001 ; \mathrm{I}^{2}=49 \%$, Figure 4 ) in paraffin-embedded tissue subgroup and SOR was $6.32(95 \%$ confidence interval 2.93 to $13.64 ; \mathrm{P}<0.00001 ; \mathrm{I}^{2}=51 \%$, Figure 4) in fresh frozen tissue subgroup.

In addition, subgroup analysis of association between HPV types and BC was conducted. In HPV16 subgroup, SOR was 6.33 (95\% confidence interval 3.47 to 11.52 ; $\mathrm{P}<0.00001 ; \mathrm{I}^{2}=10 \%$, Figure 5). In HPV18 subgroup, SOR was 3.49 (95\% confidence interval 2.24 to 5.41 ; $\mathrm{P}<0.00001 ; \mathrm{I}^{2}=0 \%$, Figure 5). In HPV33 subgroup, SOR was 3.20 (95\% confidence interval 1.64 to $2.26 ; \mathrm{P}=0.0007$, $\mathrm{I}^{2}=0 \%$, Figure 5).

\section{Publication bias and trim \& fill analysis}

We also examined the influence of publication bias through picturing a funnel graph, conducting Egger's linear regression test and Begg's rank correlation test (Figure 6). There was no publication bias found for Begg's rank correlation test $(\operatorname{Pr}>|z|=0.628)$. However, the $\mathrm{P}$ value of Egger's linear regression test was 0.003. Egger's linear regression test proved the existence of publication bias and that is the reason why trim \& fill analysis was performed. The application of the trim and fill method did not change the risk estimate (SOR 1.478, 95\% confidence interval 1.110 to 1.847 ). These data favor our previous theory that HPV infection is related to $\mathrm{BC}$.

\section{Discussion}

The results of this meta-analysis support the hypothesis put forward by Band et al., which means the HPV infection could be a potential risk factor for BC, with SOR of original case control studies as high as 6.22. The SOR of this meta-analysis is higher than that in previous meta-analysis of Bae (13) which further indicates the potential association between HPV infection and BC. The transmission path of HPV could be through blood, body contact and lymphatic fluid. The virus finally arriving the breast tissue may participate in the growth and development of tumor. Although the mechanisms involving in this biological behavior are still under discussion, we should never neglect the impact of warts infection in breast malignant neoplasm.

Considering the data in the sub group about histological type of control group, we found that an interesting fact that the SOR of normal breast tissue subgroup is lower than that in benign breast lesion subgroup. Despite the I-square value in either subgroup is under $50 \%$ which indicated heterogeneity is acceptable, the I-square value between subgroups is obvious. This discovery described the importance of control selection. Although benign breast tissues are more easily to be obtained, there are still some difference between normal breast tissue and benign breast lesion. We cannot figure the underlying reason up till now, but we boldly came up with a hypothesis that maybe in normal breast tissue the consistence infection of HPV is more dangerous than benign breast tissues. Still, whether benign breast lesion is a kind of precancerosis remains unsettled but this article indicates the scientists to avoid considering benign breast lesions as a control in future.

Regarding to the detection approaches, we noticed that more case control studies use paraffin-embedded tissue for HPV PCR detection, only 11 studies used fresh frozen tissue. Li et al. (49) have reported that HPV detection rates were slightly higher when HPV DNA was extracted from paraffin-embedded tissue than from fresh frozen tissue, which hints that biopsy taken or slide preparation may affect the detection results. However, in our subgroup analysis, 


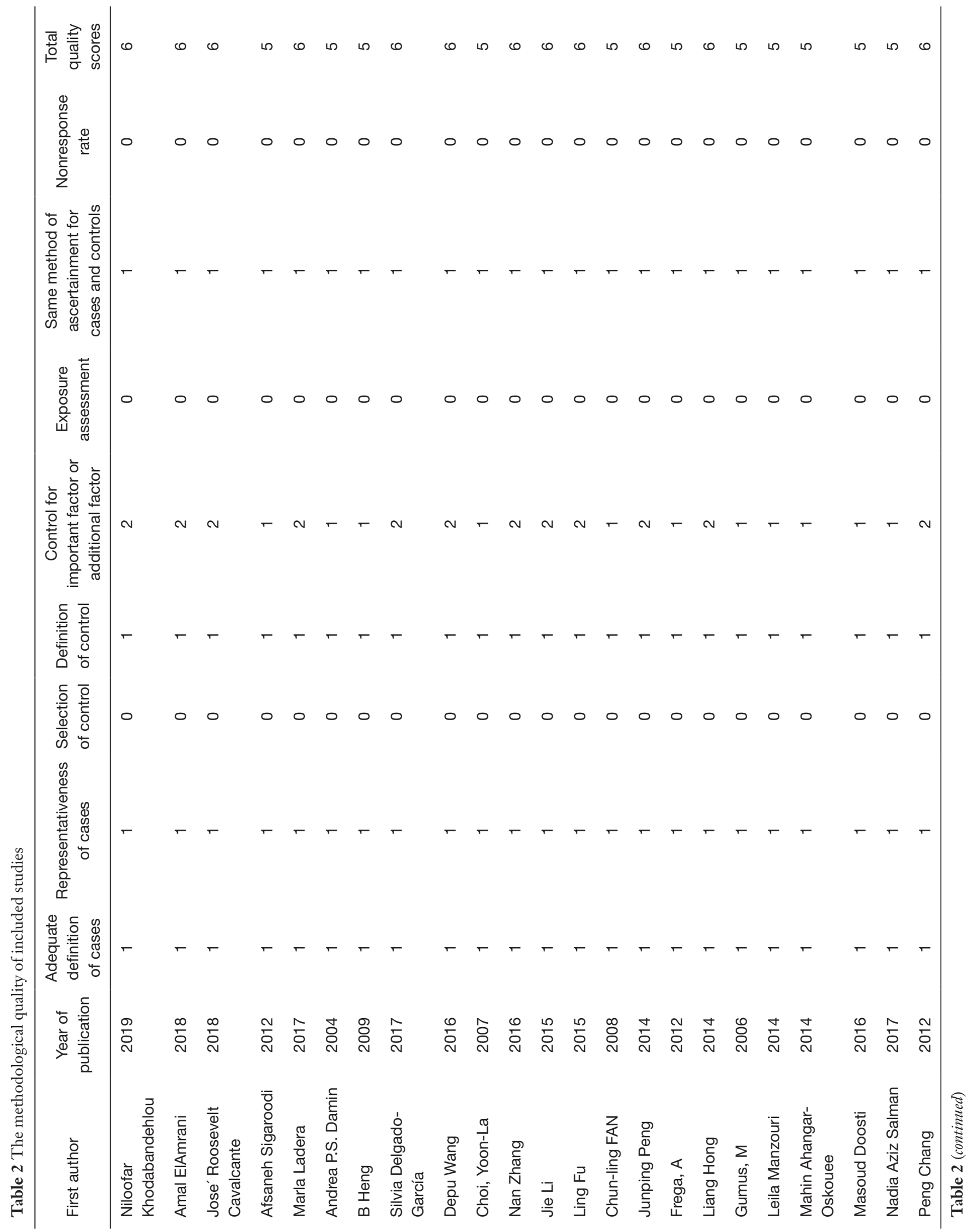




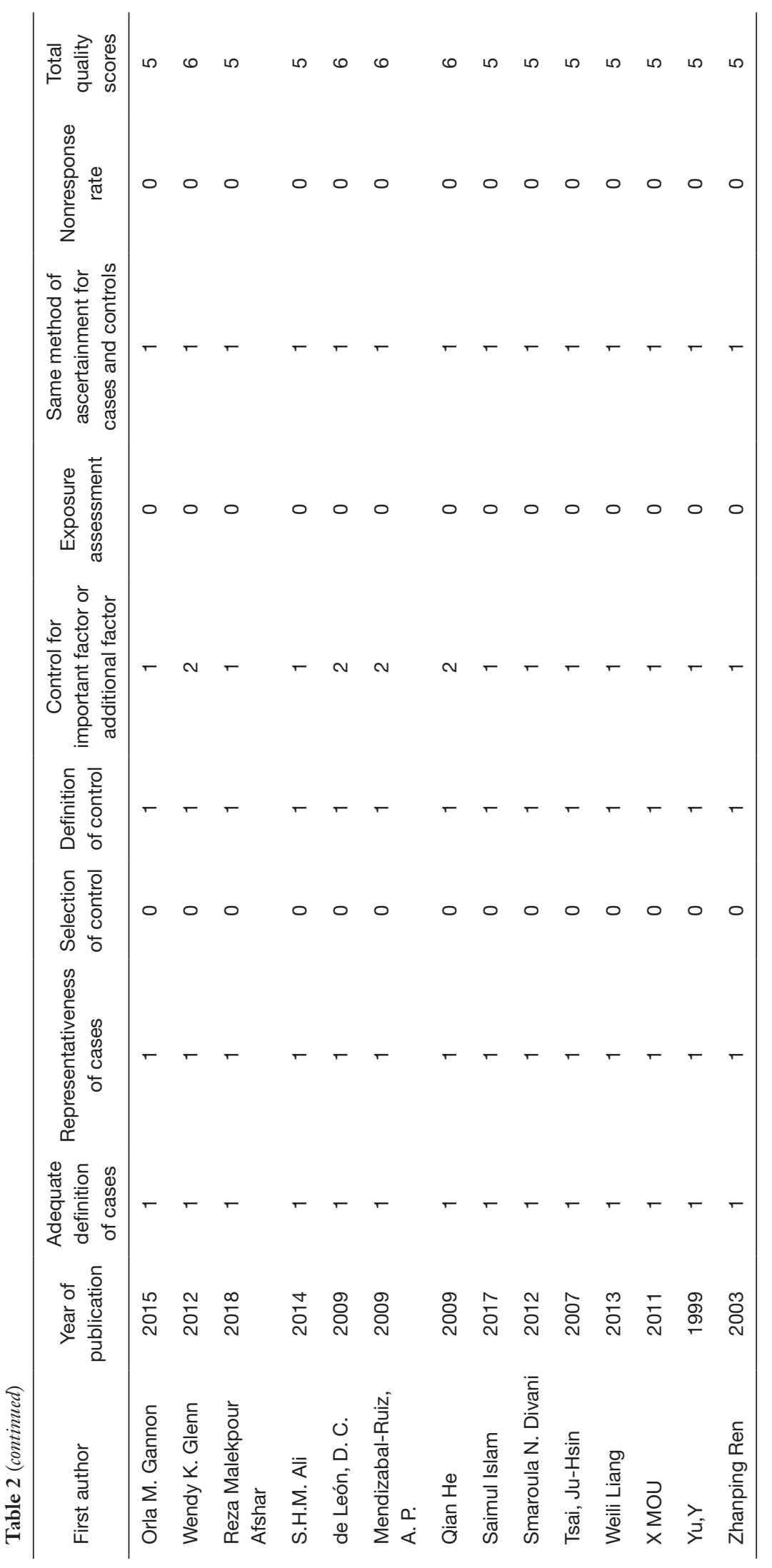




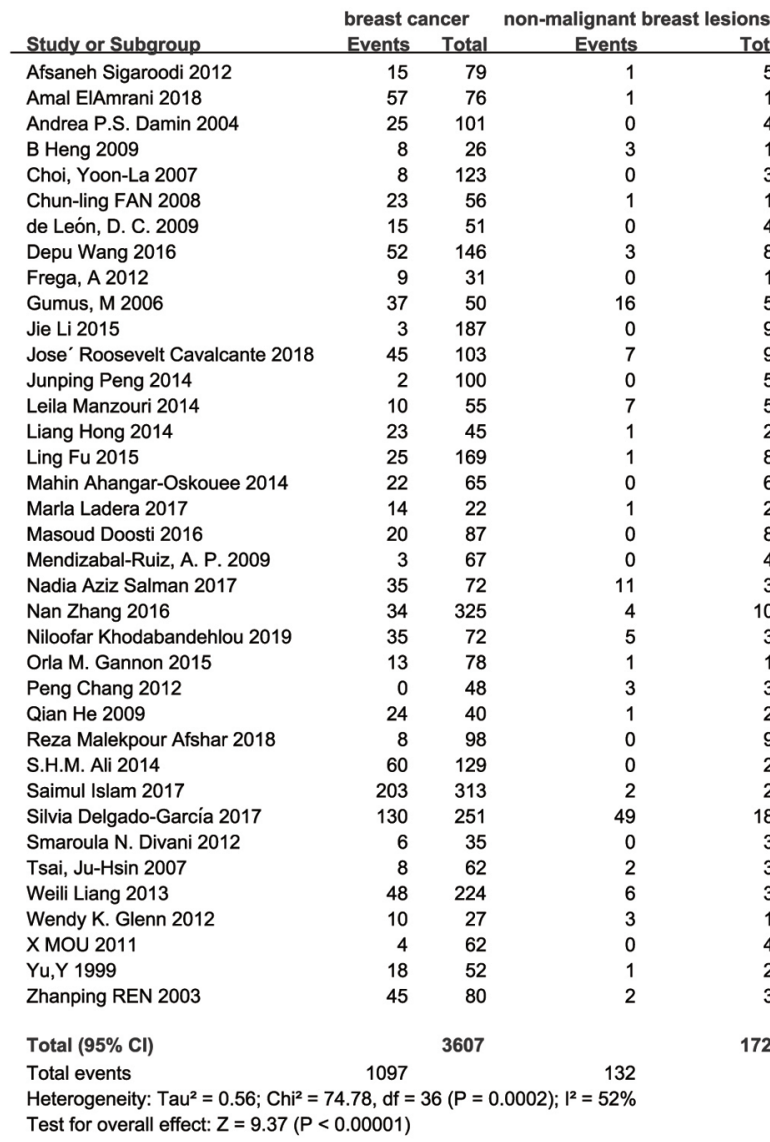

Figure 2 Forest plot of 37 included original studies.

we did not get the similar conclusion, we speculate this result may due to the heterogeneity of fresh frozen tissue subgroup $\left(I^{2}=51 \%\right)$. Besides the development of detection method may be an important influence factor of the studies. Before 2000, only type-specific PCR primers were used in the detection of HPV in breast tissues. Afterwards, broad spectrum PCR primers and the combined usage of type specific and broad-spectrum primers were used (49). The progress of technique made the detection result more stable and reliable.

HPV has over 150 subtypes. The high-risk HPV types have higher oncogenicity and cause many kinds of tumors such as cervical cancer (50) and oropharyngeal carcinoma (51). In this article, we carried out a subgroup analysis on three high-risk HPV types (HPV 16, 18 and 33). It turned out the three high-risk HPV were all related to $\mathrm{BC}$ with $\mathrm{P}$ value $<0.05$. This result is in accordance with the research of most original studies and many researches on other carcinomas.
Odds Ratio

-H, Random, $95 \% \mathrm{Cl}$

$11.72[1.50,91.74]$ $33.00[3.99,272.72]$

$27.67[1.64,466.13]$

$2.07[0.46,9.29]$

$4.64[0.26,82.53]$

$10.45[1.29,84.78]$

$36.95[2.14,638.90]$

$14.75[4.44,49.04]$

$10.56[0.57,197.02]$

$6.05[2.54,14.40]$

$3.51[0.18,68.66]$

$9.75[4.12,23.11]$

$2.56[0.12,54.41]$

$1.40[0.49,4.00]$

$19.86[2.45,161.26]$

$14.24[1.89,107.00]$

$67.76[4.00,1146.52]$

$36.75[4.13,327.09]$

$51.33[3.05,864.19]$

$4.40[0.22,87.33]$

$2.15[0.92,5.01]$

$2.80[0.97,8.11]$

$4.92[1.70,14.24]$

$1.80[0.21,15.45]$

$0.08[0.00,1.63]$

$28.50[3.46,234.61]$

$18.50[1.05,325.16]$

35.69 [2.11, 602.68]

$35.69[2.11,602.68]$
$17.53[4.01,76.66]$

$3.00[1.99,4.52]$

$15.64[0.85,289.36]$

$2.22[0.44,11.14]$

$1.41[0.56,3.57]$

$2.94[0.68,12.73]$

$7.15[0.38,136.27]$

$10.06[1.24,81.36]$

$18.00[4.01,80.75]$

$6.22[4.25,9.12]$

Odds Ratio

M-H, Random, 95\% CI
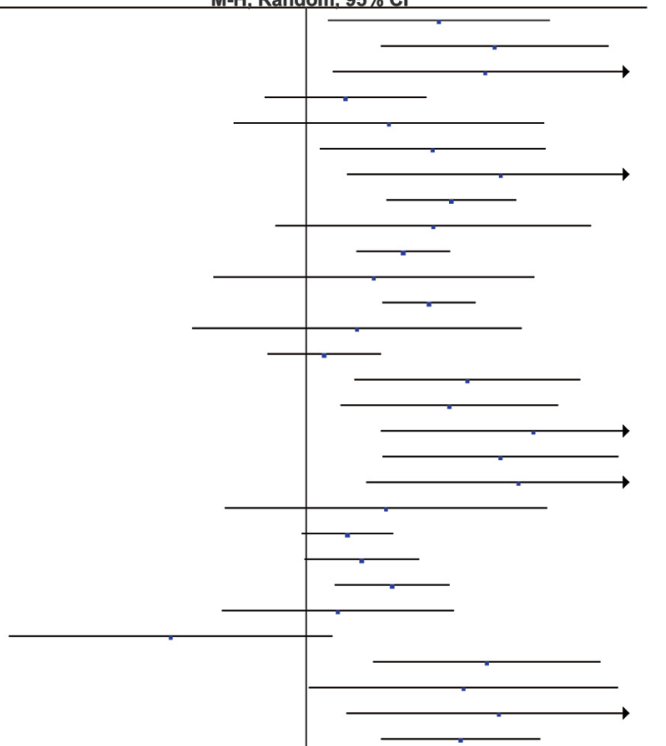

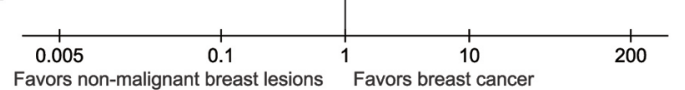

In the process of quality assessment, we found that although we included 37 original case control studies and performed a relative large-scale meta-analysis, the quality of included studies was generally low. Only 16 studies got 6 stars in terms of NOS scale. That means there is a possibility of confounding factors influencing the results to this meta-analysis, while these confounding factors could have been associated with the mechanism of HPV oncogenicity in BC, such as age, family history, menarche age, TNM stages estrogen and progesterone receptors and/ or HER2 oncogene expression, etc.

As to the potential mechanism, association between HPV and BC has not been confirmed yet. We have to admit that the appearance of HPV is not sufficient to prove virus' etiological role in $\mathrm{BC}$ development. However, the HPV infection is expected to be an early event, followed by cumulative changes over the years, similar to cervical carcinogenesis (52). Lots of theories were proposed. Khodabandehlou et al. reported that the presence of the 


\begin{tabular}{lrrrr} 
& \multicolumn{3}{c}{ breast cancer } & non-malignant breast lesion \\
Study or Subgroup & Events & Total & Events & To \\
\hline 1.2.1 normal breast tissue & & & & \\
Zhanping REN 2003 & 45 & 80 & 16 \\
Gumus, M 2006 & 37 & 50 & 7 \\
Jose' Roosevelt Cavalcante 2018 & 45 & 103 & 1 \\
Liang Hong 2014 & 23 & 45 & 5 \\
Niloofar Khodabandehlou 2019 & 35 & 72 & 1 \\
Qian He 2009 & 24 & 40 & 0 \\
S.H.M. Ali 2014 & 60 & 129 & 2 \\
Saimul Islam 2017 & 203 & 313 & 3 \\
Wendy K. Glenn 2012 & 10 & 27 & 3 \\
Subtotal (95\% Cl) & \multicolumn{3}{c}{859} & 37 \\
Total events & 482 & &
\end{tabular}

Test for overall effect: $Z=9.24(P<0.00001)$

1.2.2 benign breast lesion Afsaneh Sigaroodi 2012 Amal EIAmrani 2018 B Heng 2009

Choi, Yoon-La 2007

Chun-ling FAN 2008

de León, D. C. 2009

Depu Wang 2016

Ethel-Michele de Villiers 2005

Frega, A 2012

Jie Li 2015

Junping Peng 2014

Leila Manzouri 2014

Ling Fu 2015

Mahin Ahangar-Oskouee 2014

Marla Ladera 2017

Masoud Doosti 2016

Mendizabal-Ruiz, A. P. 2009

Nadia Aziz Salman 2017

Nan Zhang 2016

Orla M. Gannon 2015

Peng Chang 2012

Reza Malekpour Afshar 2018

Silvia Delgado-Garcia 2017

Smaroula N. Divani 2012

Tsai, Ju-Hsin 2007

Weili Liang 2013

$X$ MOU 201

Yu,Y 1999

Subtotal $(95 \% \mathrm{Cl})$

Total events

Heterogeneity: $\mathrm{Tau}^{2}=0.56 ; \mathrm{Chi}^{2}=52.05, \mathrm{df}=26(P=0.002) ; \mathrm{l}^{2}=50 \%$

Test for overall effect: $Z=6.70(P<0.00001)$

Total $(95 \% \mathrm{Cl})$

Heterogeneity: $\mathrm{Tau}^{2}=0.55 ; \mathrm{Chi}^{2}=72.82, \mathrm{df}=35(\mathrm{P}=0.0002) ; \mathrm{I}^{2}=52 \%$

Test for overall effect: $Z=9.23(P<0.00001)$

Test for subaroun differences: $\mathrm{Chi}^{2}=3.02$. df $=1(P=0.08) . \mathrm{I}^{2}=66.9 \%$

Figure 3 Subgroup analysis-histology type of control.

HPV was associated with increased inflammatory cytokines (IL-1, IL-6, IL-17, TGF- $\beta$, TNF- $\alpha$, and NF-kB) and tumor progression (15). Wang et al. revealed that HPV16 E7 may promote the proliferation of breast cancer cells by upregulating COX-2 (53). Besides, Yan et al. discovered the knockdown of HPV18 E6 and E7 could suppress the proliferation, metastasis, and cell cycle progression in HPV positive breast cancer cell line (54). What's more, Michael B. Burns' team had revealed that the mutation and deletions of DNA cytosine deaminase APOBEC3B (A3B) which functions by inhibiting retrovirus replication would elevate the risk of BC (55). After that, in 2014 Vieira and Ohba team reported the presence of HPV could alter
Odds Ratio

H, Random, $95 \%$ C M-H, Random, 95\% C

$18.00[4.01,80.75]$ $6.05[2.54,14.40]$ $9.75[4.12,23.11]$ $19.86[2.45,161.26]$ $4.92[1.70,14.24]$ $28.50[3.46,234.61]$ $35.69[2.11,602.68]$ $17.53[4.01,76.66]$ $2.94[0.68,12.73]$ $8.78[5.54,13.92]$
$11.72[1.50,91.74]$ $33.00[3.99,272.72]$ $2.07[0.46,9.29]$ $4.64[0.26,82.53]$ $10.45[1.29,84.78]$ $36.95[2.14,638.90]$ $14.75[4.44,49.04]$ $3.11[0.63,15.43]$ $10.56[0.57,197.02]$ $3.51[0.18,68.66]$ $2.56[0.12,54.41]$

$1.40[0.49,4.00]$ $14.24[1.89,107.00]$ $67.76[4.00,1146.52]$ $36.75[4.13,327.09]$ $51.33[3.05,864.19]$ $4.40[0.22,87.33]$ $2.15[0.92,5.01]$ $2.80[0.97,8.11]$ $1.80[0.21,15.45]$ $0.08[0.00,1.63]$ $18.50[1.05,325.16]$ $3.00[1.99,4.52]$
$15.64[0.85,289.36]$ $2.22[0.44,11.14]$ $1.41[0.56,3.57]$ $7.15[0.38,136.27]$ $10.06[1.24,81.36]$ $4.91[3.08,7.82]$

$6.08[4.14,8.92]$

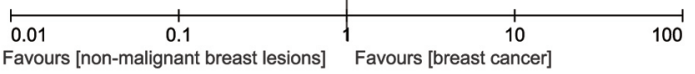

the expression of APOBEC3B (A3B) $(56,57)$. So, it is reasonable to suppose $\mathrm{HPV}$ involves in the early stage of BC by affecting APOBEC3B (A3B).

Comparing with previous systemic reviews on the similar topic, this article limited the study type as case control study instead of case only studies. Although the results of previous systemic reviews are mostly positive, due to the improvement of study design and more original researches included the conclusion in this article should be more exact and convincing. Besides, multiple subgroup analysis provide evidence for further mechanism exploration. As the debate that had been discussed by Lawson et al. in 2016, the low HPV viral load is the reason why some original 


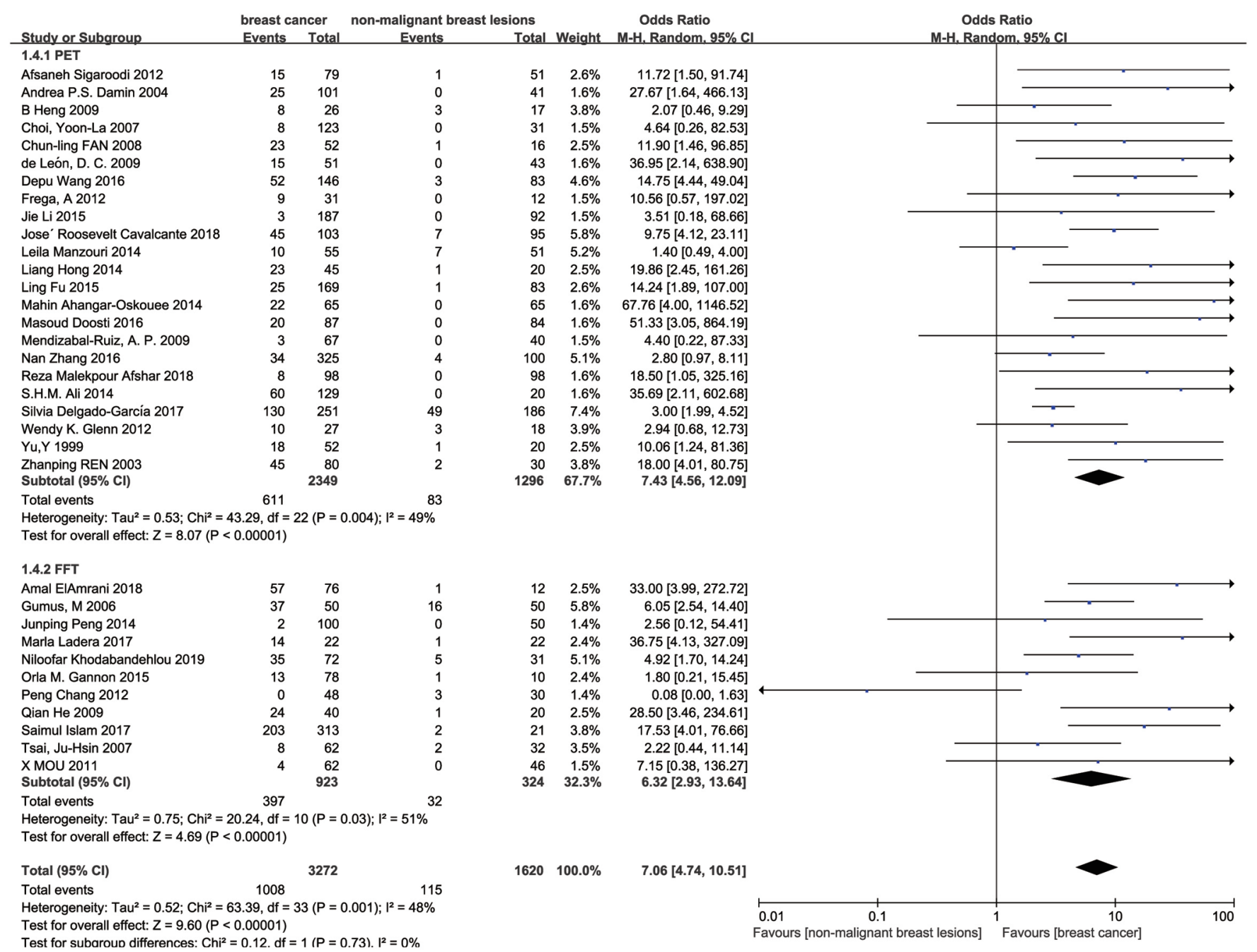

Figure 4 Subgroup analysis-tissue type.

researches obtained negative results (58). However, with the development of technique, the SOR did not become higher in most original studies within recent 5 years. We suspected that some certain kinds of BC are HPV-related while others are not.

\section{Limitations of the review}

Our meta-analysis has certain limitations. For instance, most of included studies were conducted in Asian countries. As is reported by $\mathrm{Li}$ et al. (49), although $32.42 \%$ of BC cases were $\mathrm{HPV}$-associated in Asians, only $12.91 \%$ were in Europeans. Also, the histology types were obliged to be analyzed by subgroup. However, due to the lack of specified data of clinical trials, we cannot perform them in this article. Thus, these limitations are likely to bring about bias. Besides the affection of area and pathological features, the approach of HPV affection should be advanced by identifying integrated virus DNA and free virus DNA, which is expected to provide more evidence for HPV oncogenicity mechanism in BC.

\section{Conclusions}

Through carefully data collection and analysis, we drew a conclusion that the infection of HPV can really increase the risk of $\mathrm{BC}$, especially for some high-risk types, such as HPV16, 18 and 33. The underlying molecular mechanism has not been settled yet. This calls for the scientists' attention and further exploration in the following research. 
A HPV 16

Study or Subgroup

Afsaneh Sigaroodi 2012

Andrea P.S. Damin 2004

B Heng 2009

Chun-ling FAN 2008

de León, D. C. 2009

Depu Wang 2016

Jie Li 2015

Leila Manzouri 2014

Mahin Ahangar-Oskouee 2014

Masoud Doosti 2016

Nadia Aziz Salman 2017

Niloofar Khodabandehlou 2019

Peng Chang 2012

Qian He 2009

Reza Malekpour Afshar 2018

S.H.M. Ali 2014

Smaroula N. Divani 2012

Zhanping REN 2003

breast cancer non-malignant breast lesions

Odds Ratio

Odds Ratio

$\begin{array}{rrr}\text { Events } & \text { Total } & \text { Eve } \\ 4 & 79 \\ 14 & 101 \\ 1 & 26 \\ 23 & 52 \\ 10 & 51 \\ 23 & 146 \\ 3 & 187 \\ 2 & 55 \\ 1 & 65 \\ 4 & 87 \\ 7 & 74 \\ 13 & 72 \\ 0 & 48 \\ 24 & 40 \\ 5 & 98 \\ 33 & 129 \\ 5 & 35 \\ 38 & 80\end{array}$

Total Weight

M-H. Random, 95\% C

M.H. Random. $95 \%$ Cl

Total $(95 \%$ Cl)

1425

Total events $\quad 210$

Heterogeneity: $\mathrm{Tau}^{2}=0.16 ; \mathrm{Ch}^{2}=17.87, \mathrm{df}=16(\mathrm{P}=0.33) ; \mathrm{I}^{2}=10 \%$

Test for overall effect: $Z=6.03(P<0.00001)$

$51 \quad 3.9 \% \quad 6.14[0.32,116.49$

$41 \quad 4.1 \% \quad 13.75[0.80,236.19]$

$\begin{array}{lll}17 & 3.2 \% & 2.06[0.08,53.52]\end{array}$

$16 \quad 7.1 \% \quad 11.90[1.46,96.85]$

$43 \quad 4.1 \% \quad 22.01[1.25,387.74]$

$83 \quad 4.2 \% \quad 31.78[1.90,530.40]$

$92 \quad 3.8 \% \quad 3.51[0.18,68.66]$

$51 \quad 3.6 \% \quad 4.81[0.23,102.69]$

$65 \quad 3.3 \% \quad 3.05[0.12,76.17]$

$84 \quad 3.9 \% \quad 9.11[0.48,171.83]$

$\begin{array}{lll}36 & 15.5 \% & 0.84[0.23,3.06]\end{array}$

$31 \quad 11.8 \% \quad 3.19[0.68,15.11]$

Not estimable

$20 \quad 7.1 \% \quad 28.50[3.46,234.61]$

$98 \quad 4.0 \% \quad 11.59[0.63,212.48]$

$20 \quad 4.2 \% \quad 14.23[0.84,241.86]$

$35 \quad 3.9 \% \quad 12.80[0.68,241.03]$

$30 \quad 12.5 \% \quad 12.67[2.83,56.78]$

$843100.0 \%$

$6.33[3.47,11.52]$

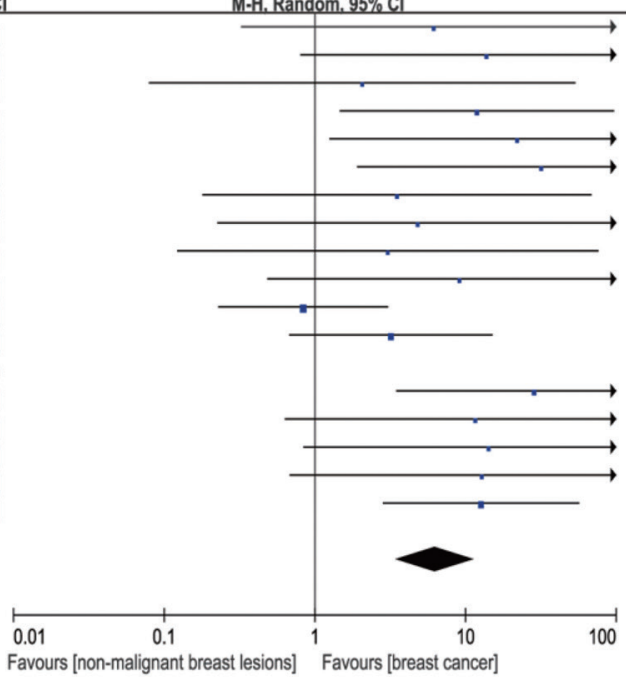

B HPV 18

breast cancer non-malignant breast lesions

Odds Ratio -H. Random. $95 \%$ C

$\begin{array}{rrr}51 & 2.2 \% & 6.14[0.32,116.49]\end{array}$

Afsaneh Sigaroodi 2012

Andrea P.S. Damin 2004

B Heng 2009

de León, D. C. 2009

Depu Wang 2016

Gumus, M 2006

Jie 12015

Jose' Roosevelt Cavalcante 2018

Juse' Roosevelt Caving Peng 2014

Leila Manzouri 2014

Masoud Doosti 2016

Nadia Aziz Salman 2017

Niloofar Khodabandehlou 2019

Peng Chang 2012

Reza Malekpour Afshar 2018

S.H.M. Ali 2014

Smaroula N. Divani 2012

Zhanping REN 2003

$\begin{array}{rrr}\text { Events } & \text { Total } & \text { Events } \\ 4 & 79 & \\ 10 & 101 & \\ 7 & 26 & \\ 3 & 51 & \\ 18 & 146 & 2 \\ 20 & 50 & \\ 2 & 187 & \\ 2 & 103 & \\ 2 & 100 & \\ 1 & 55 & \\ 3 & 87 & \\ 8 & 74 & \\ 16 & 72 & \\ 0 & 48 & \\ 5 & 98 & \\ 35 & 129 & \\ 1 & 35 & \\ 43 & 80 & 3 \\ & & \end{array}$

Total $(95 \% \mathrm{Cl})$

1521

Total events 180

$180 \quad 25$

Heterogeneity: $\mathrm{Tau}^{2}=0.00 ; \mathrm{Chi}^{2}=11$

$41 \quad 2.4 \% \quad 9.52[0.55,166.42]$

$\begin{array}{lll}17 & 8.4 \% & 1.72[0.38,7.85\end{array}$

$43 \quad 2.2 \% \quad 6.28[0.32,125.01]$

$83 \quad 8.8 \% \quad 5.70[1.29,25.20]$

$50 \quad 23.1 \% \quad 3.04[1.21,7.60]$

$92 \quad 2.1 \% \quad 2.49[0.12,52.47]$

$95 \quad 4.9 \% \quad 0.92[0.13,6.67]$

$50 \quad 2.1 \% \quad 2.56[0.12,54.41]$

$50 \quad 2.1 \%$

$51 \quad 1.9 \%$

$84 \quad 2.2 \%$

$36 \quad 10.0 \%$

$31 \quad 11.2 \%$

30

$98 \quad 2.3 \%$

$20 \quad 2.4 \%$

$\begin{array}{ll}20 & 2.4 \% \\ 35 & 1.9 \%\end{array}$

$2.83[0.11,71.18]$

$7.00[0.36,137.61]$

$1.33[0.33,5.36]$

$2.67[0.72,9.92]$

Not estimable

$11.59[0.63,212.48]$

$15.40[0.91,261.47]$

$3.09[0.12,78.41]$

$10.46[2.93,37.29]$

$937 \quad 100.0 \%$

$3.49[2.24,5.41]$

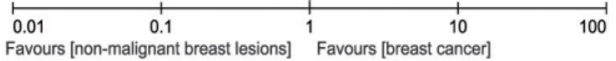

C HPV 33

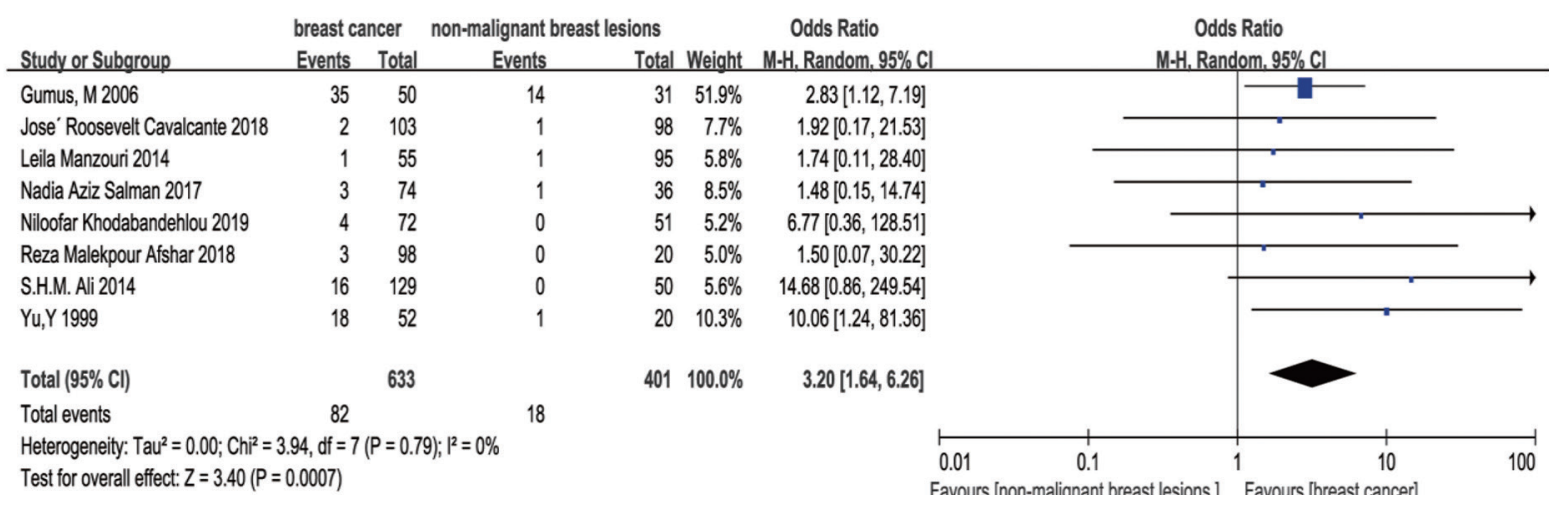

Figure 5 Forest plot of three high risk HPV types: (A) HPV16, (B) HPV18, (C) HPV33. 
A

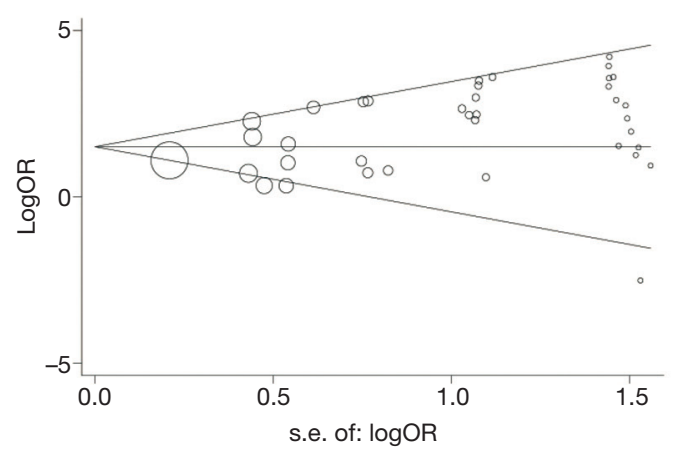

B

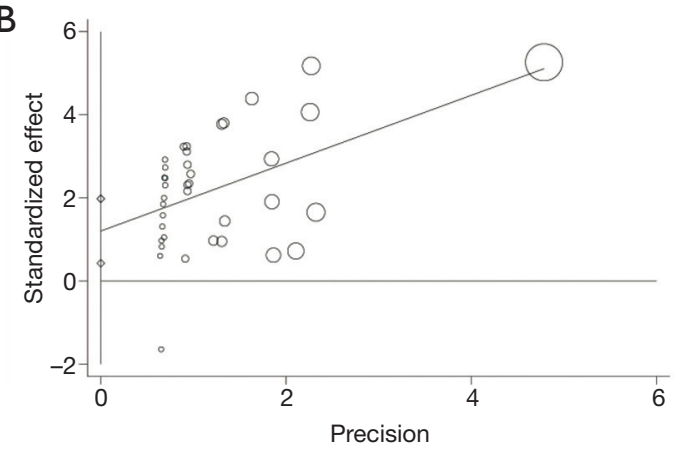

C

\begin{tabular}{ccccccc}
\hline Std_Eff & Coef. & Std. Err & $\mathrm{t}$ & $\mathrm{P}>|\mathrm{t}|$ & \multicolumn{2}{c}{ [95\% Conf. Interval] } \\
\hline slope & 0.8163713 & 0.2624402 & 3.11 & 0.004 & 0.2835893 & 1.349153 \\
Bias & 1.203004 & 0.382542 & 3.14 & 0.003 & 0.4264028 & 1.979606
\end{tabular}

D

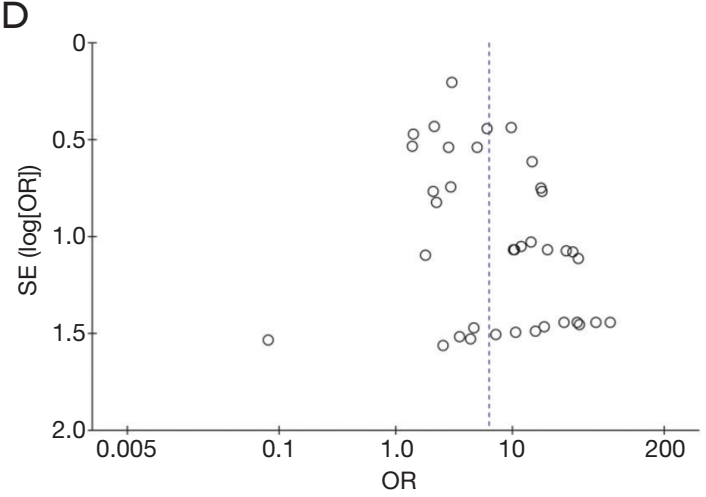

$\mathrm{E}$

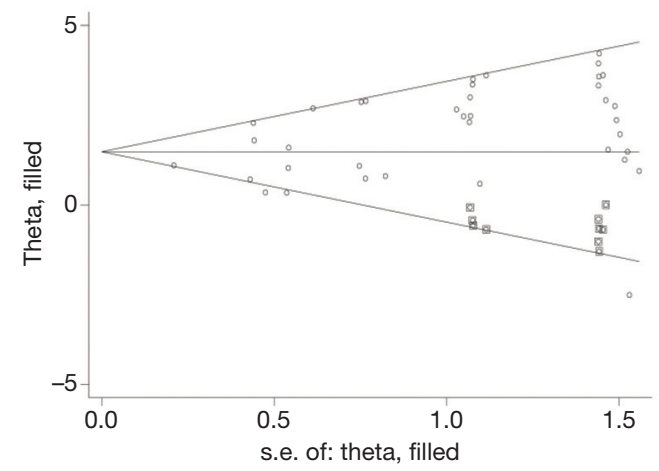

Figure 6 Publication bias evaluation: (A) Begg's funnel plot with pseudo 95\% confidence intervals; (B) Egger's publication bias plot; (C) Egger's test result; (D) Funnel plot of included studies; (E) filled funnel plot with pseudo $95 \%$ confidence intervals.

\section{Acknowledgments}

We would like to acknowledge the librarians at the Libraries of Central South University for their efforts in obtaining primary resources for this meta-analysis. Additionally, we would like to acknowledge professors, colleagues, friends and family members who assisted and gave encouragements in the writing procedure of this article.

\section{Footnote}

Conflicts of Interest: The authors have no conflicts of interest to declare.

Ethical Statement: The authors are accountable for all aspects of the work in ensuring that questions related to the accuracy or integrity of any part of the work are appropriately investigated and resolved.

\section{References}

1. DeSantis CE, Fedewa SA, Goding Sauer A, et al. Breast cancer statistics, 2015: Conver-gence of incidence rates between black and white women. CA Cancer J Clin 2016;66:31-42.

2. Bray F, Ferlay J, Soerjomataram I, et al. Global cancer statistics 2018: GLOBOCAN esti-mates of incidence and mortality worldwide for 36 cancers in 185 countries. CA Cancer J Clin 2018;68:394-424.

3. Ferrini K, Ghelfi F, Mannucci R, et al. Lifestyle, nutrition and breast cancer: facts and presumptions for consideration. Ecancermedicalscience 2015;9:557.

4. Castelló A, Martin M, Ruiz A, et al. Lower Breast Cancer Risk among Women following the World Cancer Research Fund and American Institute for Cancer Research Lifestyle Recommendations: EpiGEICAM Case-Control Study. PLoS One 2015;10:e0126096. 
5. Myers ER, McCrory DC, Nanda K, et al. Mathematical model for the natural history of human papillomavirus infection and cervical carcinogenesis. Am J Epidemiol 2000;151:1158-71.

6. Boshart M, Gissmann L, Ikenberg H, et al. A new type of papillomavirus DNA, its presence in genital cancer biopsies and in cell lines derived from cervical cancer. EMBO J 1984;3:1151-7.

7. Band V, Zajchowski D, Kulesa V, et al. Human papilloma virus DNAs immortalize normal human mammary epithelial cells and reduce their growth factor requirements. Proc Natl Acad Sci U S A 1990;87:463-7.

8. Doosti M, Bakhshesh M, Zahir ST, et al. Lack of Evidence for a Relationship between High Risk Human Papillomaviruses and Breast Cancer in Iranian Patients. Asian Pac J Cancer Prev 2016;17:4357-61.

9. Bakhtiyrizadeh S, Hosseini SY, Yaghobi R, et al. Almost Complete Lack of Human Cy-tomegalovirus and Human papillomaviruses Genome in Benign and Malignant Breast Lesions in Shiraz, Southwest of Iran. Asian Pac J Cancer Prev 2017;18:3319-24.

10. Gannon OM, Antonsson A, Milevskiy M, et al. No association between HPV positive breast cancer and expression of human papilloma viral transcripts. Sci Rep 2015;5:18081.

11. Cavalcante JR, Pinheiro LGP, Almeida PRC, et al. Association of breast cancer with human papillomavirus (HPV) infection in Northeast Brazil: molecular evidence. Clinics (Sao Paulo) 2018;73:e465.

12. Stroup DF, Berlin JA, Morton SC, et al. Meta-analysis of observational studies in epide-miology: a proposal for reporting. Meta-analysis Of Observational Studies in Epidemi-ology (MOOSE) group. JAMA 2000;283:2008-12.

13. Bae JM, Kim EH. Human papillomavirus infection and risk of breast cancer: a me-ta-analysis of case-control studies. Infect Agent Cancer 2016;11:14.

14. Simões PW, Medeiros LR, Simoes Pires PD, et al. Prevalence of human papillomavirus in breast cancer: a systematic review. Int J Gynecol Cancer 2012;22:343-7.

15. Khodabandehlou N, Mostafaei S, Etemadi A, et al. Human papilloma virus and breast cancer: the role of inflammation and viral expressed proteins. BMC Cancer 2019;19:61.

16. Malekpour Afshar R, Balar N, Mollaei HR, et al. Low Prevalence of Human Papilloma Virus in Patients with Breast Cancer, Kerman; Iran. Asian Pac J Cancer Prev 2018;19:3039-44.

17. ElAmrani A, Gheit T, Benhessou M, et al. Prevalence of mucosal and cutaneous human papillomavirus in Moroccan breast cancer. Papillomavirus Res 2018;5:150-5.

18. Salman NA, Davies G, Majidy F, et al. Association of High Risk Human Papillomavirus and Breast cancer: A UK based Study. Sci Rep 2017;7:43591.

19. Ladera M, Fernandes A, López M, et al. Presence of human papillomavirus and Ep-stein-Barr virus in breast cancer biopsies as potential risk factors. Gaceta Mexicana de Oncologia 2017;16:107-12.

20. Islam S, Dasgupta H, Roychowdhury A, et al. Study of association and molecular analysis of human papillomavirus in breast cancer of Indian patients: Clinical and prognostic implication. PLoS One 2017;12:e0172760.

21. Delgado-García S, Martínez-Escoriza JC, Alba A, et al. Presence of human papillomavirus DNA in breast cancer: A Spanish case-control study. BMC Cancer 2017;17:320.

22. Zhang N, Ma ZP, Wang J, et al. Human papillomavirus infection correlates with inflammatory Stat 3 signaling activity and IL-17 expression in patients with breast cancer. Am J Transl Res 2016;8:3214-26.

23. Wang D, Fu L, Shah W, et al. Presence of high risk HPV DNA but indolent transcription of E6/E7 oncogenes in invasive ductal carcinoma of breast. Pathol Res Pract 2016;212:1151-6.

24. Li J, Ding J, Zhai K. Detection of Human Papillomavirus DNA in Patients with Breast Tumor in China. PLoS One 2015;10:e0136050.

25. Fu L, Wang D, Shah W, et al. Association of human papillomavirus type 58 with breast cancer in Shaanxi province of China. J Med Virol 2015;87:1034-40.

26. Peng J, Wang T, Zhu H, et al. Multiplex PCR/mass spectrometry screening of biological carcinogenic agents in human mammary tumors. J Clin Virol 2014;61:255-9.

27. Manzouri L, Salehi R, Shariatpanahi S, et al. Prevalence of human papilloma virus among women with breast cancer since 2005-2009 in Isfahan. Adv Biomed Res 2014;3:75.

28. Hong L, Tang S. Does HPV 16/18 infection affect p53 expression in invasive ductal car-cinoma? An experimental study. Pak J Med Sci 2014;30:789-92.

29. Ali SHM, Al-Alwan NAS, Al-Alwany SHM. Detection and genotyping of human papil-lomavirus in breast cancer tissues from Iraqi patients. East Mediterr Health J 2014;20:372-7.

30. Ahangar-Oskouee $M$, Shahmahmoodi S, Jalilvand S, et al. No detection of 'high-risk' human papillomaviruses in a group of Iranian women with breast cancer. Asian Pac J Cancer Prev 2014;15:4061-5.

31. Liang W, Wang J, Wang C, et al. Detection of highrisk human papillomaviruses in fresh breast cancer samples using the hybrid capture 2 assay. J Med Virol 
2013;85:2087-92.

32. Sigaroodi A, Nadji SA, Naghshvar F, et al. Human papillomavirus is associated with breast cancer in the north part of Iran. ScientificWorldJournal 2012;2012:837191.

33. Glenn WK, Heng B, Delprado W, et al. Epstein-Barr virus, human papillomavirus and mouse mammary tumour virus as multiple viruses in breast cancer. PLoS One 2012;7:e48788.

34. Divani SN, Giovani AM. Detection of human papillomavirus DNA in fine needle aspirates of women with breast cancer. Arch Oncol 2012;20:12-4.

35. Chang P, Wang T, Yao Q, et al. Absence of human papillomavirus in patients with breast cancer in north-west China. Med Oncol 2012;29:521-5.

36. Frega A, Lorenzon L, Bononi M, et al. Evaluation of E6 and E7 mRNA expression in HPV DNA positive breast cancer. Eur J Gynaecol Oncol 2012;33:164-7.

37. Mou X, Chen L, Liu F, et al. Low prevalence of human papillomavirus (HPV) in Chinese patients with breast cancer. J Int Med Res 2011;39:1636-44.

38. Heng B, Glenn WK, Ye Y, et al. Human papilloma virus is associated with breast cancer. Br J Cancer 2009;101:1345-50.

39. He Q, Zhang SQ, Chu YL, et al. The correlations between HPV16 infection and expres-sions of c-erbB-2 and bcl-2 in breast carcinoma. Mol Biol Rep 2009;36:807-12.

40. Mendizabal-Ruiz AP, Morales JA, Ramirez-Jirano LJ, et al. Low frequency of human papillomavirus DNA in breast cancer tissue. Breast Cancer Res Treat 2009;114:189-94.

41. de León DC, Montiel DP, Nemcova J, et al. Human papillomavirus (HPV) in breast tumors: prevalence in a group of Mexican patients. BMC Cancer 2009;9:26.

42. Fan CL, Zhou JH, Hu CY. Expression of human papillomavirus in mammary carcinoma and its possible mechanism in carcinogenesis. Virologica Sinica 2008;23:226-31.

43. Choi YL, Cho EY, Kim JH, et al. Detection of human papillomavirus DNA by DNA chip in breast carcinomas of Korean women. Tumour Biol 2007;28:327-32.

44. Tsai JH, Hsu CS, Tsai CH, et al. Relationship between viral factors, axillary lymph node status and survival in breast cancer. J Cancer Res Clin Oncol 2007;133:13-21.

45. Gumus M, Yumuk PF, Salepci T, et al. HPV DNA frequency and subset analysis in human breast cancer patients' normal and tumoral tissue samples. J Exp Clin Cancer Res 2006;25:515-21.

46. Damin AP, Karam R, Zettler CG, et al. Evidence for an association of human papilloma-virus and breast carcinomas. Breast Cancer Res Treat 2004;84:131-7.
47. Ren Z, Huang J, Shi Z, et al. Detection of human papillomavirus types 16 and 18 infection in breast cancer tissues by Primed in situ labeling. Zhongguo Zhongliu Linchuang 2003;30:243-6.

48. Yu Y, Morimoto T, Sasa M, et al. HPV33 DNA in premalignant and malignant breast lesions in Chinese and Japanese populations. Anticancer Res 1999;19:5057-61.

49. Li N, Bi X, Zhang Y, et al. Human papillomavirus infection and sporadic breast carcinoma risk: a metaanalysis. Breast Cancer Res Treat 2011;126:515-20.

50. Auborn KJ, Woodworth C, DiPaolo JA, et al. The interaction between HPV infection and estrogen metabolism in cervical carcinogenesis. Int J Cancer 1991;49:867-9.

51. Tobouti PL, Bolt R, Radhakrishnan R, et al. Altered Tolllike receptor expression and function in HPV-associated oropharyngeal carcinoma. Oncotarget 2017;9:236-48.

52. Malhone C, Longatto-Filho A, Filassi JR. Is Human Papilloma Virus Associated with Breast Cancer? A Review of the Molecular Evidence. Acta Cytol 2018;62:166-77.

53. Wang YX, Zhang ZY, Wang JQ, et al. HPV16 E7 increases COX-2 expression and pro-motes the proliferation of breast cancer. Oncol Lett 2018;16:317-25.

54. Yan C, Teng Zhi P, Chen Yun X, et al. Viral Etiology Relationship between Human Pap-illomavirus and Human Breast Cancer and Target of Gene Therapy. Biomed Environ Sci 2016;29:331-9.

55. Burns MB, Lackey L, Carpenter MA, et al. APOBEC3B is an enzymatic source of mutation in breast cancer. Nature 2013;494:366-70.

56. Ohba $\mathrm{K}$, Ichiyama $\mathrm{K}$, Yajima $M$, et al. In vivo and in vitro studies suggest a possible in-volvement of HPV infection in the early stage of breast carcinogenesis via APOBEC3B induction. PLoS One 2014;9:e97787.

57. Vieira VC, Leonard B, White EA, et al. Human papillomavirus E6 triggers upregulation of the antiviral and cancer genomic DNA deaminase APOBEC3B. MBio 2014;5:e02234-14.

58. Lawson JS, Glenn WK, Whitaker NJ. Human Papilloma Viruses and Breast Cancer - Assessment of Causality. Front Oncol 2016;6:207.

Cite this article as: Ren C, Zeng $\mathrm{K}, \mathrm{Wu} \mathrm{C}, \mathrm{Mu} \mathrm{L}$, Huang J, Wang M. Human papillomavirus infection increases the risk of breast carcinoma: a large-scale systemic review and metaanalysis of case-control studies. Gland Surg 2019;8(5):486-500. doi: $10.21037 /$ gs.2019.09.04 


\section{Supplementary I Search strategy in Pubmed database}

(((“Papillomaviridae”[Mesh]) OR (c(c(c(c(c(“Human Papilloma Virus”[Title/Abstract)) OR “Human Papilloma Viruses”[Title/ Abstract]) OR "Papilloma Virus, Human"[Title/Abstract]) OR "Papilloma Viruses, Human"[Title/Abstract]) OR "Virus, Human Papilloma"[Title/Abstract]) OR "Viruses, Human Papilloma"[Title/Abstract]) OR "HPV, Human Papillomavirus Viruses"[Title/Abstract]) OR "Human Papillomavirus Viruses"[Title/Abstract]) OR "Human Papillomavirus Virus"[Title/ Abstract]) OR "Papillomavirus Virus, Human”[Title/Abstract]) OR "Papillomavirus Viruses, Human”[Title/Abstract]) OR "Virus, Human Papillomavirus"[Title/Abstract]) OR "Viruses, Human Papillomavirus"[Title/Abstract]))) AND (("Breast Neoplasms”[Mesh]) OR Abstract]) OR "Breast Tumors"[Title/Abstract]) OR "Breast Tumor"[Title/Abstract]) OR "Tumor, Breast”[Title/ Abstract]) OR “Tumors, Breast”[Title/Abstract]) OR “Neoplasms, Breast”[Title/Abstract]) OR "Breast Cancer”[Title/ Abstract]) OR “Cancer, Breast”[Title/Abstract]) OR “Mammary Cancer"[Title/Abstract]) OR “Cancer, Mammary"[Title/ Abstract]) OR "Cancers, Mammary"[Title/Abstract]) OR “Mammary Cancers”[Title/Abstract]) OR "Malignant Neoplasm of Breast”[Title/Abstract]) OR "Breast Malignant Neoplasm”[Title/Abstract]) OR "Breast Malignant Neoplasms”[Title/ Abstract]) OR "Malignant Tumor of Breast"[Title/Abstract]) OR "Breast Malignant Tumor"[Title/Abstract]) OR "Breast Malignant Tumors"[Title/Abstract]) OR “Cancer of Breast”[Title/Abstract]) OR “Cancer of the Breast”[Title/Abstract]) OR "Mammary Carcinoma, Human"[Title/Abstract]) OR "Carcinoma, Human Mammary"[Title/Abstract]) OR "Carcinomas, Human Mammary"[Title/Abstract]) OR "Human Mammary Carcinomas"[Title/Abstract]) OR "Mammary Carcinomas, Human"[Title/Abstract]) OR "Human Mammary Carcinoma"[Title/Abstract]) OR "Mammary Neoplasms, Human”[Title/ Abstract]) OR "Human Mammary Neoplasm”[Title/Abstract]) OR "Human Mammary Neoplasms”[Title/Abstract]) OR

"Neoplasm, Human Mammary”[Title/Abstract]) OR "Neoplasms, Human Mammary"[Title/Abstract]) OR "Mammary Neoplasm, Human"[Title/Abstract]) OR "Breast Carcinoma"[Title/Abstract]) OR "Breast Carcinomas"[Title/Abstract]) OR “Carcinoma, Breast”[Title/Abstract]) OR “Carcinomas, Breast”[Title/Abstract])) Sort by: Best Match

Table S1 MOOSE checklist for meta-analyses of observational studies

\begin{tabular}{|c|c|c|}
\hline Item No & Recommendation & Reported on page No \\
\hline \multicolumn{3}{|c|}{ Reporting of background should include } \\
\hline 1 & Problem definition & 2,3-4 \\
\hline 2 & Hypothesis statement & 2,3-4 \\
\hline 3 & Description of study outcome(s) & 2 \\
\hline 4 & Type of exposure or intervention used & 2 \\
\hline 5 & Type of study designs used & 2 \\
\hline 6 & Study population & 2 \\
\hline \multicolumn{3}{|c|}{ Reporting of search strategy should include } \\
\hline 7 & Qualifications of searchers (e.g., librarians and investigators) & N/A \\
\hline 8 & Search strategy, including time period included in the synthesis and key words & 4 \\
\hline 9 & Effort to include all available studies, including contact with authors & 4 \\
\hline 10 & Databases and registries searched & 4 \\
\hline 11 & Search software used, name and version, including special features used (eg, explosion) & $\mathrm{N} / \mathrm{A}$ \\
\hline 12 & Use of hand searching (e.g., reference lists of obtained articles) & 4 \\
\hline 13 & List of citations located and those excluded, including justification & Figure 1 \\
\hline 14 & Method of addressing articles published in languages other than English & N/A \\
\hline 15 & Method of handling abstracts and unpublished studies & 5 \\
\hline 16 & Description of any contact with authors & 5 \\
\hline \multicolumn{3}{|c|}{ Reporting of methods should include } \\
\hline 17 & $\begin{array}{l}\text { Description of relevance or appropriateness of studies assembled for assessing the hypothesis } \\
\text { to be tested }\end{array}$ & 4 \\
\hline 18 & Rationale for the selection and coding of data (e.g., sound clinical principles or convenience) & $4-5$ \\
\hline 19 & $\begin{array}{l}\text { Documentation of how data were classified and coded (e.g., multiple raters, blinding and } \\
\text { interrater reliability) }\end{array}$ & $4-5$ \\
\hline 20 & $\begin{array}{l}\text { Assessment of confounding (e.g., comparability of cases and controls in studies where } \\
\text { appropriate) }\end{array}$ & Table 2 \\
\hline 21 & $\begin{array}{l}\text { Assessment of study quality, including blinding of quality assessors, stratification or regression } \\
\text { on possible predictors of study results }\end{array}$ & Table 2 \\
\hline 22 & Assessment of heterogeneity & Figure1 \\
\hline 23 & $\begin{array}{l}\text { Description of statistical methods (e.g., complete description of fixed or random effects models, } \\
\text { justification of whether the chosen models account for predictors of study results, dose-response } \\
\text { models, or cumulative meta-analysis) in sufficient detail to be replicated }\end{array}$ & 5 \\
\hline 24 & Provision of appropriate tables and graphics & Figures 2-6 \\
\hline \multicolumn{3}{|c|}{ Reporting of results should include } \\
\hline 25 & Graphic summarizing individual study estimates and overall estimate & Figure 1 \\
\hline 26 & Table giving descriptive information for each study included & Table 1 \\
\hline 27 & Results of sensitivity testing (e.g., subgroup analysis) & 6 \\
\hline 28 & Indication of statistical uncertainty of findings & $6-7$ \\
\hline \multicolumn{3}{|c|}{ Reporting of discussion should include } \\
\hline 29 & Quantitative assessment of bias (e.g., publication bias) & $6-7$ \\
\hline 30 & Justification for exclusion (e.g., exclusion of non-English language citations) & 4 \\
\hline 31 & Assessment of quality of included studies & 8 \\
\hline \multicolumn{3}{|c|}{ Reporting of conclusions should include } \\
\hline 32 & Consideration of alternative explanations for observed results & 9 \\
\hline 33 & $\begin{array}{l}\text { Generalization of the conclusions (i.e., appropriate for the data presented and within the domain } \\
\text { of the literature review) }\end{array}$ & 9 \\
\hline 34 & Guidelines for future research & 9 \\
\hline 35 & Disclosure of funding source & 9 \\
\hline
\end{tabular}

From: Stroup DF, Berlin JA, Morton SC, et al. for the Meta-analysis Of Observational Studies in Epidemiology (MOOSE) Group. Meta-analysis of Observational Studies in Epidemiology. A Proposal for Reporting. JAMA. 2000;283(15):2008-2012. doi: 10.1001/ jama.283.15.2008. Transcribed from the original paper within the NEUROSURGERY ${ }^{\circledR}$ Editorial Office, Atlanta, GA, United Sates. August 2012. 